\title{
Improving the Quality of Service in Wireless Body Area Networks using Genetic Algorithm
}

\author{
${ }^{1}$ Mr.Benaka Santhosha S, ${ }^{2}$ Mrs.B.R.Sujatha \\ ${ }^{1}$ Dept of ECE. MCE, HassanKarnataka, India \\ ${ }^{2}$ Associate Prof: Dept of ECE. MCE, Hassan Karnataka, India
}

\begin{abstract}
Wireless Body Area Sensor Networks are the highly distributed network, in which its nodes are the organizer itself and each of them has the flexibility of gathering and sending patient biomedical knowledge to a terminal, that referred to as sink. When knowledge sent to sink from a path that doesn't have a definite basis, the routing is a crucial challenge in Wireless Body Area Sensor Networks, additionally reliability and routing delay are the considerable factors in these varieties of networks. A replacement methodology for improvement of routing that is using Genetic Algorithm has been proposed in this paper, such that for each of mentioned factors, a weight primarily based on their importance is specified, that are repeated over the races and have improved the reliability and delay. Analysis and assess of proposed algorithm simulation ends up in MATLAB, giving a motivating improvement than alternative typical ways.
\end{abstract}

\section{Keywords; Wireless Body Area Network(WBAN),Genetic Algorithm(GA), Delay, Routing, Quality of Service.}

\section{INTRODUCTION}

Recent technological advances in sensors, low power microelectronics and miniaturization, and wireless networking enabled the design and proliferation of wireless sensor networks, capable of autonomously monitoring and controlling environments. One of the most promising applications of sensor networks is for human health monitoring. A number of tiny wireless sensors, strategically placed on the human body, create a wireless body area network (WBAN) [1], which can monitor various vital signs, providing real time feedback to the user and medical personnel. Patients or non-patients will not only be able to get medical advice from a distance, but also be able to send from any location full, detailed, and accurate vital signal measurements, as if they had been taken in a medical center, termed as the "ubiquitous medical care". WBAN can be considered as a special type of sensor network with its own specific requirements. WBAN consists of a set of mobile and compact intercommunicating sensors, either wearable or implanted into the human body, which monitor vital body parameters and movements. These devices, communicating through wireless technologies, transmit data from the body to a home base station, from where the data can be forwarded to a hospital, clinic, or elsewhere in real time [2]. In Wireless Body Area Sensor Networks, each node includes three systems. First, sensor subsystem that senses the data. Second, process subsystem that performs the processing and third, connection subsystem that has the responsibility of sending data between neighboring nodes. This network includes many sensor nodes that are widespread in the environment, gathers the data and has the ability to connect each other and also to outer base station. Sensor nodes have the limited sensing capacity and processing in the limited region.

Some of the technological requirements of WBANs are the use of a broadband signaling scheme, frequency range below 6
$\mathrm{GHz}$, and the support of heterogeneous multi-hop links to cope with the high path loss through the human body.

The wireless body area network (WBAN) is a resource constraint network in terms of energy, storage, computational capacity, buffer and bandwidth. The faulty design of routing protocol may exhaust all these resources, and may lead to frequent death of the nodes. This reduces the lifetime of the network and leads to poor Quality of service (QoS) i.e. increased drop and delay, with decreased throughput by consuming optimized resources. Therefore designing a protocol for achieving better quality of service in WBAN is required. There are various parameters which need to be addressed in the problem to formulate a solution such as:

- Dynamic Topology: In WBAN, network topology dynamically changes because of various reasons such as node mobility, interferences because of physical obstacles, node death due to technical fault or zero battery etc. Therefore, a dynamic optimization process is required for handling complexities posed by dynamic topology. So a well suited mobility model need to be considered. Random way point mobility model is one of the most suitable mobility model to be considered in WBAN.

- Optimized utilization of Energy: The routing in WBAN follows multi-hop and multipath. Therefore many control messages will be unicasted and broadcasted to establish a best suitable path in dynamic environment, which may lead to more consumption of energy as well as congestion, which may also leads to the reduced QoS. Therefore a proper mechanism is required for best path selection in search space. Genetic Algorithm is being used as search optimizing technology, which is an evolutionary algorithm and most suitable for heuristic based solution space. 
- Routing in multi-sink scenario: WBAN is being conceptualized for human safety and monitoring their health conditions. It may adopt multi sink data gathering mechanism for different parameters. Therefore data prioritization should be considered while routing to the right sink, without compromising with the QoS.

- Issues of heterogeneity: There is a non uniformity in power management, memory and processing in WBAN, which causes several challenges, which need to be handled to achieve better QoS.

Therefore to design a reliable routing protocol with optimal usage of energy, it should consider the delivery of data from the source, only when requested periodically on the occurrence of an event. Therefore a weight should be assigned to every service, and an optimum path should be chosen based on the weight of the service, to deliver the data to the sink by consuming minimum energy and maintaining higher QoS.

\section{RELATED WORK}

Carmen C. Y et.al [3] explores the use of this conduit in the security mechanism of BASN; that is, by a biometrics approach that uses an intrinsic characteristic of the human body as the authentication identity or the means of securing the distribution of a cipher key to secure inter-BASN communications.

Pervez Khan et.al [4] presents a comprehensive discussion on the applications of WBANs in smart healthcare systems. They highlight a number of projects that enable WBANs to provide unobtrusive long-term healthcare monitoring with real-time updates to the health center. They list many potential medical applications of a WBAN including epileptic seizure warning, glucose monitoring, and cancer detection.

Shuo Xiao et.al [5] investigates the dynamic adaptation of radio transmit power as a means of addressing this challenge. Their contributions are categorized into three-fold: they present empirical evidence that wireless link quality in body area networks changes rapidly when patients move; fixed radio transmit power therefore leads to either high loss (when link quality is bad), or wasted energy (when link quality is good).

Chris Otto et.al [6] present hardware and software architecture of a working wireless sensor network system for ambulatory health status monitoring. The system consists of multiple sensor nodes that monitor body motion and heart activity, a network coordinator, and a personal server running on a personal digital assistant or a personal computer.

Khalid Abu Al-Saud et.al [7] conclude that life-saving applications, studies and tests should be conducted before WBANs are widely applied to humans, particularly to address the challenges related to robust techniques for detection and classification to increase the accuracy and hence the confidence of applying such techniques without physician intervention.

Ahmed Faheem [8] presents some of the basic issues such as designing, protocols and social issues about the Wireless Body Area Networks, which have to be considered for an effective implementation of WBAN.

\section{Proposed SySTEM}

The main objective of the project work is to implement a new method for the improvement of routing and reliability, with decreased delay, in WBAN using Genetic Algorithm. Genetic Algorithms are the family of computational models inspired by evolution. These algorithms encode a potential solution to a specific problem on a simple chromosome-like data structure and apply recombination operators to these structures to preserve critical information. Genetic algorithms are often viewed as function optimizer. In this case, for each of mentioned factors such as reliability, routing and so on, a weight based on their importance is specified, to improve the reliability and to decrease the delay. The simulation model of the framework for reliable routing in wireless body area network will have following key concept to be implemented:

- Network Model: A random way point model is considered for the mobility model and sensor nodes are deployed in distributed manner.

- Assignment of Weight: The priority of data delivery in WBAN depend upon critical event, therefore the data routing will happen based on the request. To classify the services it will be assigned or labeled a weight.

For reliability: $60 \%$

For delay: $40 \%$

- Chromosome: The most challenging task is to design a chromosome, where the population of chromosomes will be a path from source to destination considering all the probable nodes with its reliability and delay by proper encoding technique. Encoding of chromosomes is one of the problem, when one started to solve problem with GA. Encoding depends on the problem. The permutation encoding will be used here to formulate the chromosome.

As the Permutation encoding can be used in ordering problems, and it is well tested method for task ordering problem. In permutation encoding, every chromosome is a string of numbers, which represents number in a sequence

\begin{tabular}{|l|llllllllll|}
\hline Chromosome A & 1 & 5 & 3 & 2 & 6 & 4 & 7 & 9 & 8 \\
\hline Chromosome B & 8 & 5 & 6 & 7 & 2 & 3 & 1 & 4 & 9 \\
\hline
\end{tabular}

- Selection of Chromosome: The suitable chromosome will be selected based on suitable fitness function and further by crossover and mutation process. It will be included into next population of generation and finally the process will continue till the objective function is achieved. The fitness function will be selected as per following equation Fitness $(\mathrm{s})=\left[\mathrm{W}_{\text {cost }} x \operatorname{Cost}(\mathrm{s})\right]+\left[\mathrm{W}_{\text {time }} x \operatorname{Time}(\mathrm{s})\right]+\left[\mathrm{W}_{\text {Rel }} x\right.$ reliability(s)]

There might be situation where because of exceptional behavior of crossover and mutation process. The fitness function might be higher than the standard value; a penalty function will be applied to keep the value within the limit. The proposed project flow can be visualized as shown in Figure 1. 


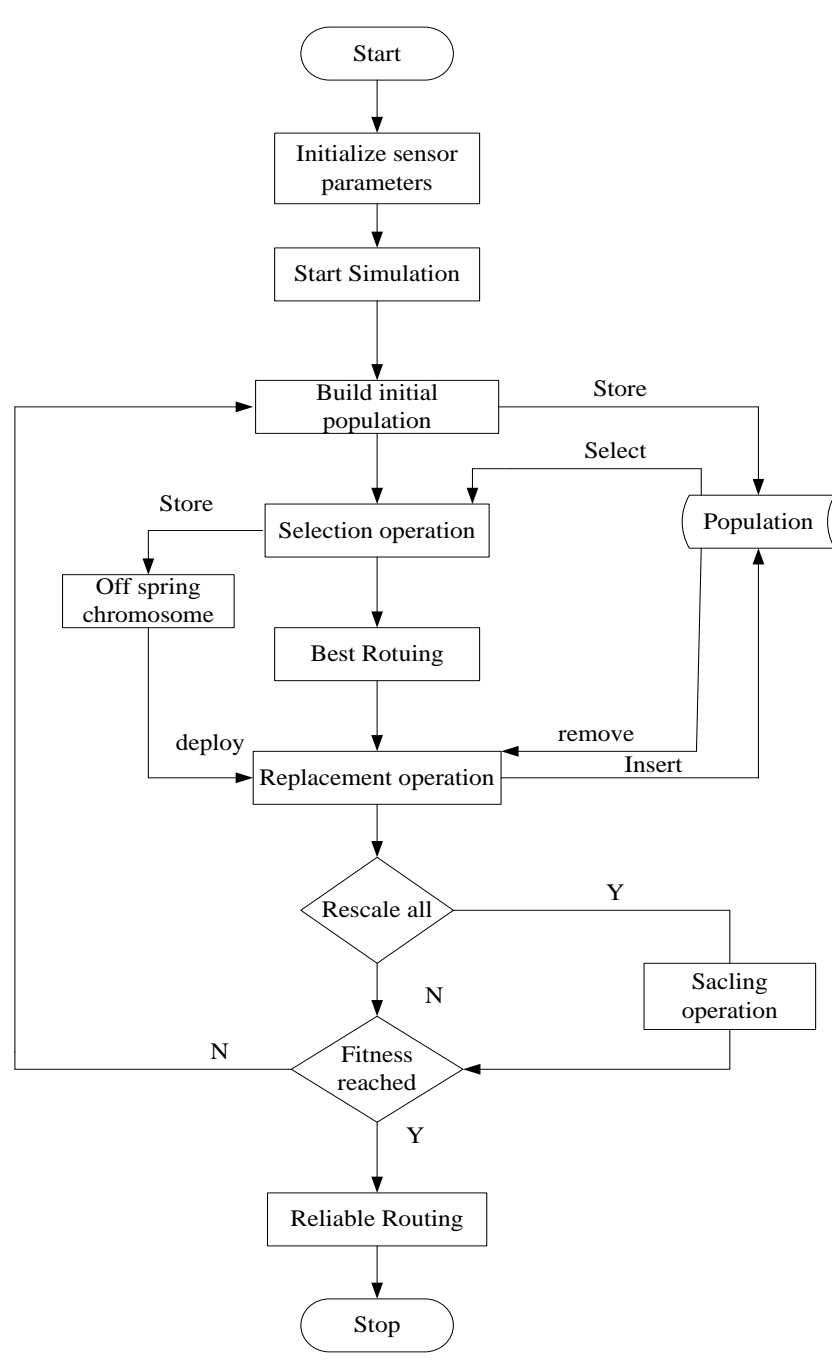

Fig. 1 Proposed Flow of project

Data aggregation or fusion is a useful technique that combines data from different sensors and avoid redundant transmissions. Our GA based approach generates a transmission schedule which consists of collection of transmission rounds. A transmission schedule denotes how data is collected from each sensor and propagated to base station. It represents a collection of routing paths that network will follow to maximize lifetime. Clearly, a routing path forms a tree that spans all the sensor nodes. To achieve fault tolerance and to extend network lifetime, relay nodes can be used for balanced data gathering. Relay nodes are some special nodes which can be used as cluster heads in hierarchical sensor networks. They are the nodes having higher energy as compared to the sensor nodes. In a two-tiered network architecture, where high powered relay nodes act as cluster heads and sensor nodes transmit their data directly to their respective cluster heads. However, the relay nodes are still battery operated and hence, power constrained. Total depletion of the power of a relay node severely impacts the functionality of the network, since all sensor nodes belonging to the cluster of the depleted relay node, they will be unable to send their data to the base station and the entire cluster becomes inoperative. This may also put additional load on the surviving relay nodes and will cause faster depletion of the batteries of other relay nodes. The algorithm used for the proposed system is as follows:

Algorithm name: Implementing GA for estimating reliability in WBAN.

Input: Node_ID, Trans_Rng, routing_param

Output: reliable routes

Steps:

1. [Start] Generate random population of $\mathrm{n}$ chromosomes (suitable solutions for the problem).

2. [Fitness] Evaluate the fitness $f(x)$ of each chromosome $x$ in the population.

3. [New population] Create a new population by repeating following steps until the new population is complete.

3.1 [Selection] Select two parent chromosomes from a population according to their fitness (the better fitness, the bigger chance to be selected)

3.2. [Crossover] with a crossover probability cross over the parents to form a new offspring (children). If no crossover was performed, offspring is an exact copy of parents.

3.3. [Mutation] with a mutation probability mutate new offspring at each locus (position in chromosome).

3.4 [Accepting] Place new offspring in a new population

4. [Replace] Use new generated population for a further run of algorithm.

5. [Test] If the end conditions are satisfied stop, and return the best solution in current population.

6. [Loop] Go to step 2.

\section{RESUlt AnAlysis}

The proposed project work is designed on Windows OS of 32 bit, $1.84 \mathrm{GHz}$ Pentium processor. Matlab environment has been used to design the simulation. The simulation area has considered around 200 nodes with multiple sink nodes. The main objective of the simulation is to highlight that using genetic algorithm; the routing protocol can be efficiently built. The proposed design is created in much cost effective manner, which can be seen with reliable figures as shown below. 


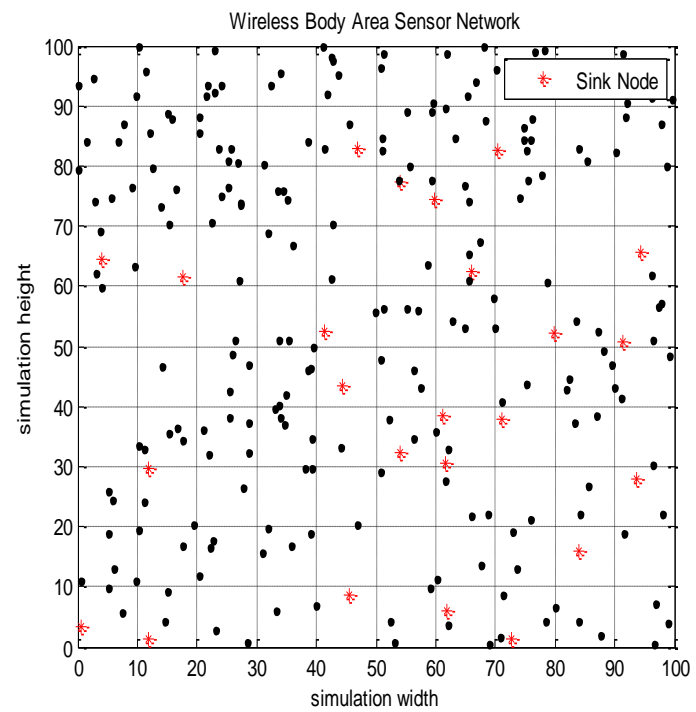

Fig 2: Delay: Simulation showing Sink Nodes and other normal nodes

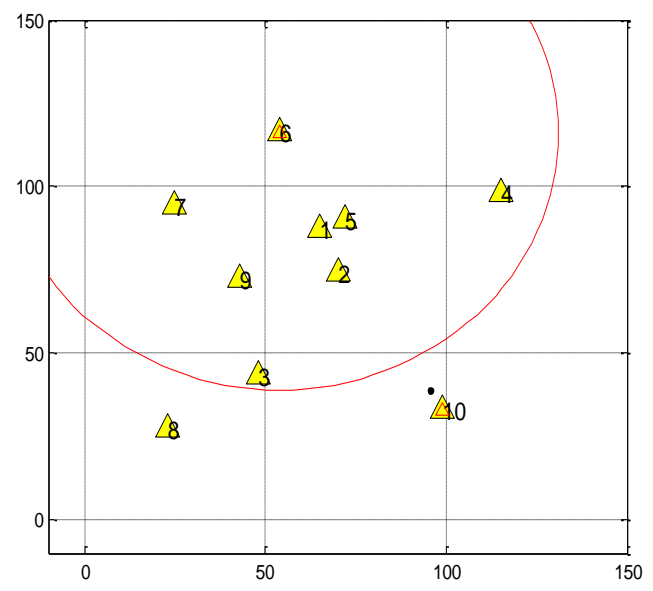

Fig 3: Visualize transmission

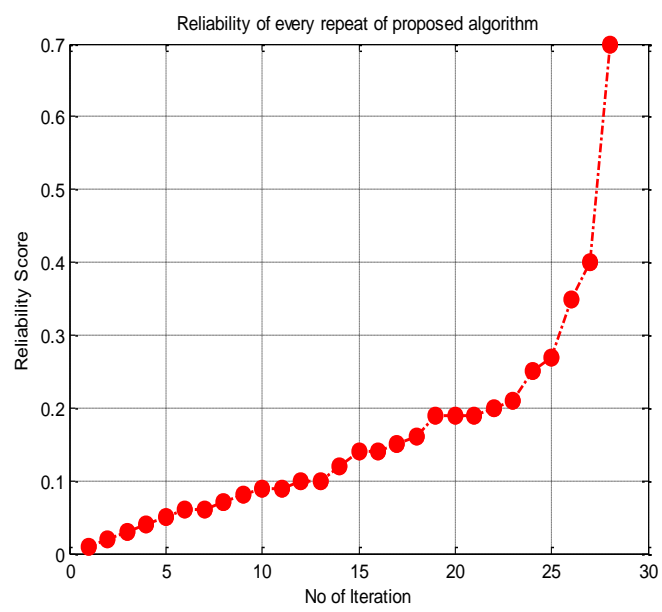

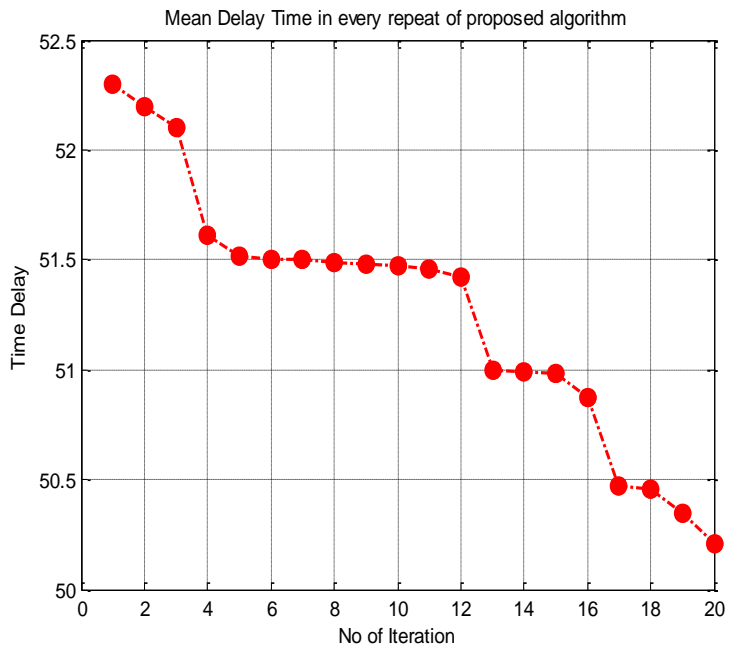

Fig 5: Mean delay time in every repeat of proposed algorithm

In this result part, we compared our proposed algorithm with the algorithms of paper [1], which the result of proposed algorithm shown is optimum result than existing algorithms [1], above diagrams show this result. Diagram 1 shows the relaibility of proposed algorithm in various repeats that reliability in every repeat is optimum. Diagram 2 shows the delay time in every repeat that delay time in every repeat is less.

\section{CONCLUSION}

A WBAN is expected to be a very useful technology with potential to offer a wide range of benefits to patients, medical personnel and society through continuous monitoring and early detection of possible problems. With the current technological evolution, sensors and radios will soon be applied as skin patches. Doing so, the sensors will seamlessly be integrated in a WBAN. Step by step, these evolutions will bring us closer to a fully operational WBAN that acts as an enabler for improving the Quality of Life. The proposed system has highlighted a routing algorithm for wireless body area sensor networks, which maximizes the reliability and decrease the delay of routing from source to destination. We feel that this review can be considered as a source of inspiration for future research directions

Fig 4: Reliability in every repeat of proposed algorithm 
IOSR Journal of Engineering

June. 2012, Vol. 2(6) pp: 1291-1295

\section{REFERENCES}

[1] S.HassanPour, B.Asadi, Y.Vejdanparast and P.Zagar "Improving Reliability of Routing in Wireless Body Area Networks", IEEE International Conference on Computer Science and Automation Engineering(CSAE), 590$593,2011$.

[2] Attaphongse Taparugssanagorn, Carlos Pomalaza-Raez, Ari Isola, Raffaello Tesi, Matti Hamalainen, Jari Iinatti "UWB Channel for Wireless Body Area Networks at Hospital" International Journal of Ultra Wideband Communications and Systems 2010 - Vol. 1, No.4 pp. $226-236$

[3] Carmen C.Y.Poon, Yuan-Ting Zhang, Shu-Di Bao"A Novel Biometrics Method to Secure Wireless Body Area Sensor Networks for Telemedicine and M-Health" IEEE Communications Magazine, Vol. 44, No. 4. (Apr 2006), pp. 73-81, doi:10.1109/MCOM.2006.1632652 Key: citeulike: 4460693

[4] Pervez Khan, Md.Asdaque Hussain, Kyung Sup Kwak "Medical Applications of Wireless Body Area Networks" International Journal of Digital Content Technology and its Applications Volume 3, Number 3, September 2009

[5] Shuo Xiao1, Vijay Sivaraman1, Alison Burdett2 "Adapting Radio Transmit Power in Wireless Body Area Sensor Networks" 3rd International ICST Conference on Body Area Networks Published 16th May 2010, Modified 16th May 2010

[6] Chris Otto, Aleksandar Milenković, Corey Sanders, Emil Jovanov "System Architecture of A Wireless Body Area Sensor Network for Ubiquitous Health Monitoring" Journal of Mobile Multimedia, Vol. 1, No.4 (2006) 307326

[7] Khalid Abu Al-Saud, Massudi Mahmuddin and Amr Mohamed "Wireless Body Area Sensor Networks Signal Processing and Communication Framework: Survey on Sensing, Communication Technologies, Delivery and Feedback" Journal of Computer Science 8 (1): 121-132, 2012 ISSN 1549-3636 @ 2012 Science Publications

[8] Ahmed Faheem "Wireless Body Area Sensor Network" Consumer Communications and Networking Conference, 2007. CCNC 2007. 4th IEEE, Jan. Page(s): 722 - 726 